\title{
Molecular Gas and the Nuclear Star Cluster in IC342: Sufficient Inflow for Recurring Star Formation Events?
}

\author{
Eva Schinnerer ${ }^{1,4}$, Torsten Böker ${ }^{2,5}$ \& David S. Meier ${ }^{3}$
}

\begin{abstract}
We present high spatial resolution $\left(1.2^{\prime \prime}\right)$ mm-interferometric observations of the ${ }^{12} \mathrm{CO}(2-1)$ line emission in the central $300 \mathrm{pc}$ of the late-type spiral galaxy IC 342. The data, obtained with the Owens Valley Radio Observatory, allow firsttime detection of a molecular gas disk that coincides with the luminous young stellar cluster in the nucleus of IC 342. The nuclear CO disk has a diameter of $\sim 30$ pc and a molecular gas mass of $\mathrm{M}_{\mathrm{H}_{2}} \sim 2 \times 10^{5} \mathrm{M}_{\odot}$. It connects via two faint $\mathrm{CO}$ bridges to the well-known, $100 \mathrm{pc}$ diameter circumnuclear gas ring. Analysis of the gas kinematics shows that the line-of-nodes in the inner $15 \mathrm{pc}$ is offset by about $45^{\circ}$ from the major kinematic axis, indicating non-circular motion of the gas within a few parsec of the dynamical center of IC 342. Both the morphology and the kinematics of the $\mathrm{CO}$ gas indicate ongoing inflow of molecular gas into the central few parsec of IC 342. We infer a gas inflow rate between 0.003 and $0.14 \mathrm{M}_{\odot} \mathrm{yr}^{-1}$, based on the observed surface density of the nuclear gas disk and estimates of the radial velocities of the surrounding gas. Inflow rates of this order can support repetitive star formation events in the nucleus of IC 342 on timescales much smaller than a Hubble time.
\end{abstract}

Subject headings: galaxies: nuclei — galaxies: ISM — galaxies: kinematics and dynamics — galaxies: individual(IC 342)

\section{INTRODUCTION}

Recent observations with the Hubble Space Telescope (HST) at both optical and nearinfrared wavelengths (e.g. Carollo et al. 1998; Böker et al. 2002) have revealed that the

\footnotetext{
${ }^{1}$ National Radio Astronomy Observatory, P.O. Box 0, Socorro, NM 87801

${ }^{2}$ Space Telescope Science Institute, 3700 San Martin Drive, Baltimore, MD 21218, U.S.A.

${ }^{3}$ University of Illinois, Urbana-Champaign, 1002 W. Green St., Urbana, IL 61801

${ }^{4}$ Jansky-Fellow

${ }^{5}$ On assignment from the Space Telescope Division of the European Space Agency (ESA).
} 
very centers of late-type spiral galaxies are often occupied by a photometrically distinct, luminous, and compact (with a few parsec diameter) stellar cluster. Most of the nuclear star clusters studied in detail appear to have experienced recent star formation. For example, M 33 (Gordon et al. 1999), NGC 4449 (Böker et al. 2001), as well as NGC 247 and NGC 2403 (Davidge \& Courteau 2002) all have nuclear clusters with a spectral energy distribution (SED) that is dominated by a young $(\lesssim 100 \mathrm{Myr})$ stellar population. Clearly, these galaxies must recently have experienced significant inflow of molecular gas into their central few parsec in order to trigger the formation of these young clusters. There is mounting evidence that large-scale bars can efficiently move large quantities of molecular gas down to the inner kiloparsec (Sakamoto et al. 1999). However, the exact mechanism which can transport gas from a few hundred parsec down to a few parsec is still under debate.

IC 342 is a prime example of a late-type spiral galaxy with a young luminous nuclear cluster which formed in a short-lived burst about 60 Myr ago (Böker, Förster-Schreiber, \& Genzel 1997; Böker, van der Marel, \& Vacca 1999). For this paper, we adopt the distance of $1.8 \mathrm{Mpc}$ derived by McCall (1989) which makes IC 342 the closest late-type galaxy with strong nuclear star formation ${ }^{6}$. It thus offers a unique opportunity to study the related gas dynamics on scales of a few parsec $\left(1^{\prime \prime} \sim 8.7 \mathrm{pc}\right)$. The central region of IC 342 has been mapped in the past in several molecular transitions on scales of a few arcseconds (e.g. Lo et al. 1984; Ishizuki et al. 1990; Turner \& Hurt 1992; Turner et al. 1993; Meier et al. 2000; Meier \& Turner 2001). These maps show that the molecular gas is located in two spiral arms which show strong streaming motions. The spiral arms join to form a ring of about $10^{\prime \prime}$ diameter which is the site of massive star formation (Böker et al. 1997). In this Letter, we present a high resolution, high sensitivity ${ }^{12} \mathrm{CO}(2-1)$ map which for the first time resolves the molecular gas kinematics inside the starburst ring.

\section{OBSERVATIONS}

The center of IC 342 was observed in the ${ }^{12} \mathrm{CO}(2-1)$ line in January 2002 with the six-element Owens Valley Radio Observatory (OVRO) millimeter interferometer. Obtained in the $\mathrm{L}$ and $\mathrm{H}$ configurations with baselines between 15 and $115 \mathrm{~m}$, the data have a spatial resolution of $\sim 1.2^{\prime \prime}(10 \mathrm{pc})$ with robust weighting. The noise per $2.601 \mathrm{~km} \mathrm{~s}^{-1}$ wide channel is $40 \mathrm{mJy}$ per beam in the combined data of both tracks. For the intensity map, we used only emission that is $5 \sigma$ above the noise floor and evident in at least two adjacent channels.

\footnotetext{
${ }^{6}$ The distance to IC 342 is uncertain, e.g. more recent observations indicate a larger distance of $3.3 \mathrm{Mpc}$ (Saha, Claver, \& Hoessel 2002). This should be kept in mind when discussing distance-dependent quantities.
} 
Similarly, we used a $15 \sigma$ blanking for the velocity field to emphasize the small-scale velocity structure. Nevertheless, our data recover most of the CO flux in the region mapped, as the peak integrated intensity of our data convolved to $14^{\prime \prime}$ resolution is about $90 \%$ of the value of $\mathrm{I}_{30 \mathrm{~m}}=324 \mathrm{~K} \mathrm{~km} \mathrm{~s}^{-1}$ measured with the IRAM 30m single dish telescope (Eckart et al. 1990). The absolute astrometry of the OVRO observations are tied to the VLA quasar reference frame, which are known to $\leq 0.02^{\prime \prime}$. However, the positional uncertainty in the observations is limited by atmospheric refraction at $230 \mathrm{GHz}$ and is $<0.1^{\prime \prime}$.

For comparison to the CO data, we retrieved Hubble Space Telescope V-band (F555W) and $\mathrm{H} \alpha$ (+continuum, F658N) maps from the newly available WFPC2 associations archive. In order to register the HST images and the CO maps, we aligned the eastern CO spiral arm to the prominent dust lane seen in both HST images. Incidentally, this also aligned the central CO peak with the nuclear star cluster. The alignment required a nominal shift of $1.28^{\prime \prime}$ to the west and $0.59^{\prime \prime}$ to the north ${ }^{7}$. Potential discrepancies of this magnitude between HST and radio coordinate systems are not uncommon (e.g. Whitmore \& Zhang 2002).

\section{MOLECULAR GAS PROPERTIES}

CO Distribution and Geometry: The intensity map in Figure 1 partly resolves the structure of the two well-known molecular spiral arms (e.g. Lo et al. 1984; Turner \& Hurt 1992; Meier et al. 2000; Meier \& Turner 2001). The two arms have quite different morphologies: the eastern arm forms a continuous spiral which is barely resolved with our data. A second, weaker component about $5^{\prime \prime}$ east of the main arm is also apparent in the map. The western arm, on the other hand, has two main components: a southern part pointing toward the nucleus, and a northern part (north of $-3^{\prime \prime}, 0^{\prime \prime}$ ) which seems to form another spiral. The western arm is about $5^{\prime \prime}$ wide and has a sharp inner edge, but becomes more diffuse toward larger radii. The mean gas density within the spiral arms is $\sim 10^{23} \mathrm{~cm}^{-2}$, assuming an intrinsic line ratio of $I_{21} / I_{10}=1$ and the standard Galactic conversion factor of $2 \times 10^{20} \mathrm{~cm}^{-2}\left(\mathrm{~K} \mathrm{~km} \mathrm{~s}^{-1}\right)^{-1}$ (Strong et al. 1988). These values translate into an average gas surface densities of $\sim 1100 \mathrm{M}_{\odot} / \mathrm{pc}^{2}$ (including a $36 \%$ contribution from He), with peak values about twice as high. The map reveals somewhat weaker emission inside the central trough that has been identified for the first time. Because this emission component has a disk-like morphology and appears centered on the nuclear star cluster (see below), we refer to it as the nuclear disk for the remainder of this paper. The disk has a major axis diameter

\footnotetext{
${ }^{7}$ The HST coordinates were measured from the positions of 8 stars in the field of view that are listed in the USNO-A2.0 catalog.
} 
of $\sim 30 \mathrm{pc}$ and a gas mass of $\sim 1.7 \times 10^{5} \mathrm{M}_{\odot}$, with a mean gas density about half of that in the spiral arms. The disk appears connected to both spiral arms via two faint CO "bridges" $\sim 1^{\prime \prime}$ north and south of the nucleus.

CO Kinematics: The overall motion of the molecular gas is from southwest to northeast, in agreement with the position angle of the kinematic major axis of $\sim 37^{\circ}$ determined from HI data (Crosthwaite et al. 2001). The arms of the gas spiral show strong streaming motions, and are interpreted as gas lanes along the leading side of a weak stellar bar (Turner \& Hurt 1992; Sakamoto et al. 1999). On smaller scales, the CO velocity field within the spiral arms (Fig. 1) does not appear well ordered since the line emission consists of multiple components. The high spatial and spectral resolution of the OVRO data allows us to identify non-circular gas motions close to the center of IC 342. The molecular gas is moving along a position angle of $\sim-10^{\circ}$ (Figs. 1 and 2) which is offset by $\sim 45^{\circ}$ from the kinematic major axis of the galaxy disk. This is the first time that non-circular motions within $10 \mathrm{pc}$ of the nucleus have been detected in IC 342, their nature is discussed further in section 4. Within the spatial resolution of our observations, the central CO peak coincides with the dynamical center as well as the nuclear star cluster, and the systemic velocity we measure is $v_{\text {LSR }} \approx 46.0 \mathrm{~km} \mathrm{~s}^{-1}$. This value is $\sim 7 \mathrm{~km} \mathrm{~s}^{-1}$ higher than inferred from lower resolution $\mathrm{CO}(1-0)$ data ( $\sim 39 \mathrm{~km} \mathrm{~s}^{-1}$ Sakamoto et al. 1999). This discrepancy is likely due to the noncircular nature of the nuclear CO kinematics which only becomes more apparent at high angular resolution.

Comparison to Optical Morphology: The distribution of the molecular gas differs from that of the stellar inventory as demonstrated by the comparison of the HST images to the $\mathrm{CO}$ distribution (Fig. 3). The CO spiral arms outline the bright central emission region that is evident in both the $\mathrm{H} \alpha$ emission and the $\mathrm{V}$-band continuum. Both the line emission and the optical continuum are brighter east of the nucleus where the CO emission is confined to the prominent dust lane. In addition, optical light fills the gap between the two eastern gas lanes. On the other hand, west of the nucleus where the CO spiral is much wider, there is a lack of $\mathrm{H} \alpha$ emission and optical continuum. This asymmetry in the extinction pattern together with the velocity field suggests that the western side is the near side of IC 342. A prominent $\mathrm{H} \alpha$ knot lies on the boundary $\left(-3^{\prime \prime} ;-1^{\prime \prime}\right)$ between the two parts of the western gas spiral, it coincides with Region 1 studied by Böker et al. (1997) and is the site of intense star formation. Within the nuclear disk, the peak of the molecular gas (which is close to the dynamical center, see $\S 3$ ) coincides roughly with the nuclear star cluster. Two other star clusters north and south of the nucleus fall into regions inside the spiral arms which are almost devoid of molecular line emission. The HST V-band image shows two fine dust lanes of $\sim 0.2^{\prime \prime}$ width about $1^{\prime \prime}$ north and south of the nucleus which extend in east-west direction 
(best seen in Fig. 2). The two CO bridges that connect the nuclear disk to the spiral arms appear to coincide with these dust lanes, although the lower spatial resolution of the CO map makes this identification somewhat tentative.

\section{DISCUSSION}

What Drives the Gas Inflow? The overall shape and kinematics of the CO spiral arms are reminiscent of models for gas flows in a weakly barred potential (e.g. Athanassoula 1992). Although no stellar bar is obvious in the optical data, ellipse fits to deep wide-field CCD images show evidence for a bar with $600-700^{\prime \prime}$ length and a position angle of $\sim 20^{\circ}$ (Buta \& McCall 1999). Since the western side is the near side (see $\S 3$ ), the sense of rotation is counter-clockwise, and therefore the gas spiral arms are on the leading side of this largescale bar. Crosthwaite et al. (2001) interpret the displacement of the optical and dynamical position angles and the warped outer HI disk as signs of a recent mild interaction. This interaction might have triggered the formation of the oval distortion which in turn might be responsible for the pile-up of molecular gas in the central kiloparsec of IC 342. The molecular gas morphology of IC 342 is quite similar to the one observed in the center of our own Galaxy where $\mathrm{a} \sim 14 \mathrm{pc}$ wide molecular disk or torus is surrounded by a molecular ring with a radius of $180 \mathrm{pc}$ (see review by Morris \& Serabyn 1996). Also, the bar length of $\sim 5.2 \mathrm{kpc}$ in IC 342 is comparable to the $4.8 \mathrm{kpc}$ observed in our Galaxy. The filamentary structure of the dust/gas lanes and the $\mathrm{H} \alpha$ emission in IC 342 (similar filaments are also observed in the inner 500 pc of our Galaxy) might be explained by self-gravitating gas (see Fig. 2 of Wada \& Koda 2001). The coincidence of the CO disk's central velocity with systemic velocity favors a scenario in which the gas lies within the disk, as opposed to being situated above the plane. The in-plane picture is supported by the good agreement between the gas distribution and the dust morphology.

In the following we will discuss three plausible interpretations of the CO morphology. First, the nuclear $\mathrm{CO}$ disk could be tilted relative to the large stellar disk. The axis ratio of the nuclear disk of $b / a \sim 0.5$ translates into an inclination of $i \sim 60^{\circ}$ along a position angle of $\sim 15^{\circ}$. The two CO peaks about $0.5^{\prime \prime}$ north and south of the center are separated in velocity by $8 \mathrm{~km} \mathrm{~s}^{-1}$ (see Fig. 2). Assuming solid body rotation and that these two peaks fall on the kinematic major axis of the nuclear disk, the resulting dynamical mass is $M_{\text {dyn }} \sim 2 \times 10^{4} \mathrm{M}_{\odot}$. The derived mass is significantly smaller than the mass estimated for either the nuclear molecular gas disk or the nuclear stellar cluster $\left(6 \times 10^{6} \mathrm{M}_{\odot}\right.$, Böker et al. 1999) making this an unlikely scenario. A second alternative is that the nuclear CO emission originates in a virialized cloud, rather than a rotating disk. For a cloud size of about $2^{\prime \prime}(17 \mathrm{pc})$ and a $\mathrm{CO}$ line width (FWHM) of $\Delta v \sim 8 \mathrm{~km} \mathrm{~s}^{-1}$, the virialized mass is 
$M_{\text {vir }} \sim 3 \times 10^{5} \mathrm{M}_{\odot}$. However, the observed rotation (see Fig. 2) within the nuclear disk argues against a single virialized cloud. In a third scenario, the observed non-circular motion are due to radial motions of material moving toward the center similar to what is observed in the central region of our Galaxy (Morris \& Serabyn 1996). Since the first two scenarios seem unlikely, we conclude that streaming motions are the most plausible explanation for the non-circular motions observed in the CO kinematics. A possible cause for such streaming motion could be the large-scale stellar bar. This scenario should be investigated further by dynamical modeling.

Feeding the nuclear star cluster: The nuclear star cluster in IC 342 has a mass of $6 \times 10^{6} \mathrm{M}_{\odot}$ and experienced a major burst of star formation $\sim 60 \mathrm{Myr}$ ago (Böker et al. 1999). The population of stars produced in this event dominates the SED of the cluster, and thus makes it difficult to quantify the contribution from possible older generation(s) of stars. As discussed in Böker et al. (1999), up to $90 \%$ of the cluster mass could have been produced in a burst that occurred 10 Gyr before the most recent one. While this number is definitely an upper limit because the described scenario lies at the extreme end of possible star formation histories, it is reasonable to assume that previous nuclear starburst events have indeed occurred. For the moment, we will assume that the most recent burst produced $30 \%$ of the cluster mass, i.e. about $2 \times 10^{6} \mathrm{M}_{\odot}$. Assuming a star formation efficiency of $20 \%$ (Elmegreen et al. 1999), about $10^{7} \mathrm{M}_{\odot}$ of molecular gas are needed in order to produce such a starburst event. This is 50 times more than the present-day mass of the nuclear CO disk $\left(M_{\mathrm{H}_{2}} \sim 1.7 \times 10^{5} \mathrm{M}_{\odot}\right)$. In what follows, we use our CO observations to constrain the inflow rate of molecular gas and to estimate how long it might take before another such starburst event can occur.

A lower limit can be calculated by assuming that it took the full 60 Myr since the last starburst for the nuclear $\mathrm{CO}$ disk to form. In this case, the inflow rate is $\sim 0.003 \mathrm{M}_{\odot} / \mathrm{yr}$ or about 3 Gyr are needed before a new starburst event could occur. We derive an upper limit by taking the observed gas surface density of $\Sigma \sim 500 \mathrm{M}_{\odot} / \mathrm{pc}^{2}$ together with the width of the two dust lanes seen in the $\mathrm{V}$-band image $\left(\sim 0.2^{\prime \prime}\right)$. This yields an estimate of the gas inflow rate onto the nuclear disk of $\sim 2000 \mathrm{M}_{\odot} / \mathrm{pc} \times v_{\mathrm{rad}}$. The inflow velocity of the gas, $v_{\text {rad }}$ can be estimated by comparing the observed circular velocity in the nuclear disk $\left(v_{\mathrm{obs}} \approx 25 \mathrm{~km} \mathrm{~s}^{-1}\right.$ at a radius of $0.5^{\prime \prime}$ or $4.4 \mathrm{pc}$ ) to the maximum expected circular velocity around the nuclear star cluster (assuming solid body rotation), $v_{\mathrm{sb}} \approx 75 \mathrm{~km} \mathrm{~s}^{-1}$. This implies that radial motions of up to $v_{\text {rad }} \sim 70 \mathrm{~km} \mathrm{~s}^{-1}$ can be present in the molecular gas next to the nuclear cluster, depending on the exact shape of the gravitational potential. These rough estimates result in an inflow rate of $\sim 0.14 \mathrm{M}_{\odot} / \mathrm{yr}$. Such an inflow rate would accumulate about $10^{7} \mathrm{M}_{\odot}$ of molecular gas in the nucleus within $70 \mathrm{Myr}$. These two estimates probably bracket the actual inflow rate, but a number of uncertainties remain. For one, the $\mathrm{H}_{2}$ mass in the center 
of IC 342 might be overestimated by a factor of 4-5 due to the use of a Galactic conversion factor, as discussed in Meier \& Turner (2001). In addition, the non-circular motions can be overestimated due to the unknown shape of the underlying gravitational potential. Nevertheless, the inflow rates discussed here are not unreasonable to support repetitive nuclear starbursts in IC 342. For example, an accumulation of $\sim 0.01 \mathrm{M}_{\odot} / \mathrm{yr}$ is sufficient to support a starburst duty cycle of about $1 \mathrm{Gyr}$. The duty cycle might even be shorter if the star formation efficiency is higher than the 20\% assumed here. Indeed, efficiencies up to $50 \%$ have been inferred for giant molecular clouds in the spiral arms of IC 342 (Meier \& Turner 2001). Moreover, the inflow rate is likely to vary over time with lower inflow rates in the past, and could increase in the future as more mass is accumulated at the center. For example, assuming a constant rate of $0.003 \mathrm{M}_{\odot} / \mathrm{yr}$ (our lower limit) over a Hubble time $\left(10^{10} \mathrm{yr}\right.$ ) results in a mass accumulation of $\sim 3 \times 10^{7} M_{\odot}$ which is larger than the dynamical mass of $\sim 2 \times 10^{7} M_{\odot}$ derived for the central 120 pc (Turner \& Hurt 1992).

Our analysis of new high-resolution maps of the ${ }^{12} \mathrm{CO}(2-1)$ emission in the center of IC 342 has revealed the following main results. We detect molecular gas coinciding with the nuclear stellar cluster. This, and the presence of streaming motions close to the nucleus, suggests that gaseous matter is currently accumulating at the nucleus of IC 342. These findings provide support for in-situ formation of nuclear clusters and repetitive nuclear starbursts. Rough estimates of gas inflow rates suggest that massive $\left(10^{6} \mathrm{M}_{\odot}\right)$ nuclear starbursts in IC 342 can be supported with duty cycles between a few hundred Myrs and 1 Gyrs.

We thank J. Turner and W. Maciejewski for helpful comments. DSM acknowledges support from the Laboratory of Astronomical Imaging at the University of Illinois.

\section{REFERENCES}

Athanassoula, E., 1992, MNRAS, 259, 345

Böker, T., Förster-Schreiber, N. M., \& Genzel, R. 1997, AJ, 114, 1883

Böker, T., van der Marel, R. P., \& Vacca, W. D. 1999, AJ, 118, 831

Böker, T., van der Marel, R. P., Mazzuca, L., Rix, H.-W., Rudnick, G., Ho, L., \& Shields, J. C. 2001, AJ, 121, 1473

Böker, T., Laine, S., van der Marel, R. P., Sarzi, M., Rix, H.-W., Ho, L., \& Shields, J. C. 2002, AJ, 123, 1389

Buta, R. J. \& McCall, M. L. 1999, ApJS, 124, 33 
Carollo, C. M., Stiavelli, M., \& Mack, J. 1998, AJ, 116, 68

Crosthwaite, L. P., Turner, J. L., Hurt, R. L., Levine, D. A., Martin, R. N., \& Ho, P. T. P. 2001, AJ, 122, 797

Davidge, T. J. \& Courteau, S. 2002, AJ, 123, 1438

Eckart, A., Downes, D., Genzel, R., Harris, A. I., Jaffe, D. T., \& Wild, W. 1990, ApJ, 348, 434

Elmegreen, B.G., Efremov, Y., Pudritz, R.E., Zinnecker, H. 1999, in "Protostars and Planets IV", Univ. of Arizona Press, p. 179

Gordon, K. D., Hanson, M.M., Clayton, G. C., Rieke, G. H., \& Misselt, K. A. 1999, ApJ, 519,165

Ishizuki, S., Kawabe, R., Ishiguro, M., Okumura, S. K., \& Morita, K. 1990, Nature, 344, 224

Lo, K. Y., et al. 1984, ApJ, 282, L59

McCall, M. L. 1989, AJ, 97, 1341

Meier, D. S., Turner, J. L., \& Hurt, R. L. 2000, ApJ, 531, 200

Meier, D. S. \& Turner, J. L. 2001, ApJ, 551, 687

Morris, M. \& Serabyn, E. 1996, ARA\&A, 34, 645

Saha, A., Claver, J., \& Hoessel, J. G. 2002, AJ, 124, 839

Sakamoto, K., Okumura, S. K., Ishizuki, S., \& Scoville, N. Z. 1999, ApJS, 124, 403

Strong, A. W., et al. 1988, A\&A, 207, 1

Turner, J. L. \& Hurt, R. L. 1992, ApJ, 384, 72

Turner, J. L., Hurt, R. L., \& Hudson, D. Y. 1993, ApJ, 413, L19

Wada, K. \& Koda, J. 2001, PASJ, 53, 1163

Whitmore, B. C. \& Zhang, Q. 2002, AJ, 124, 1418 

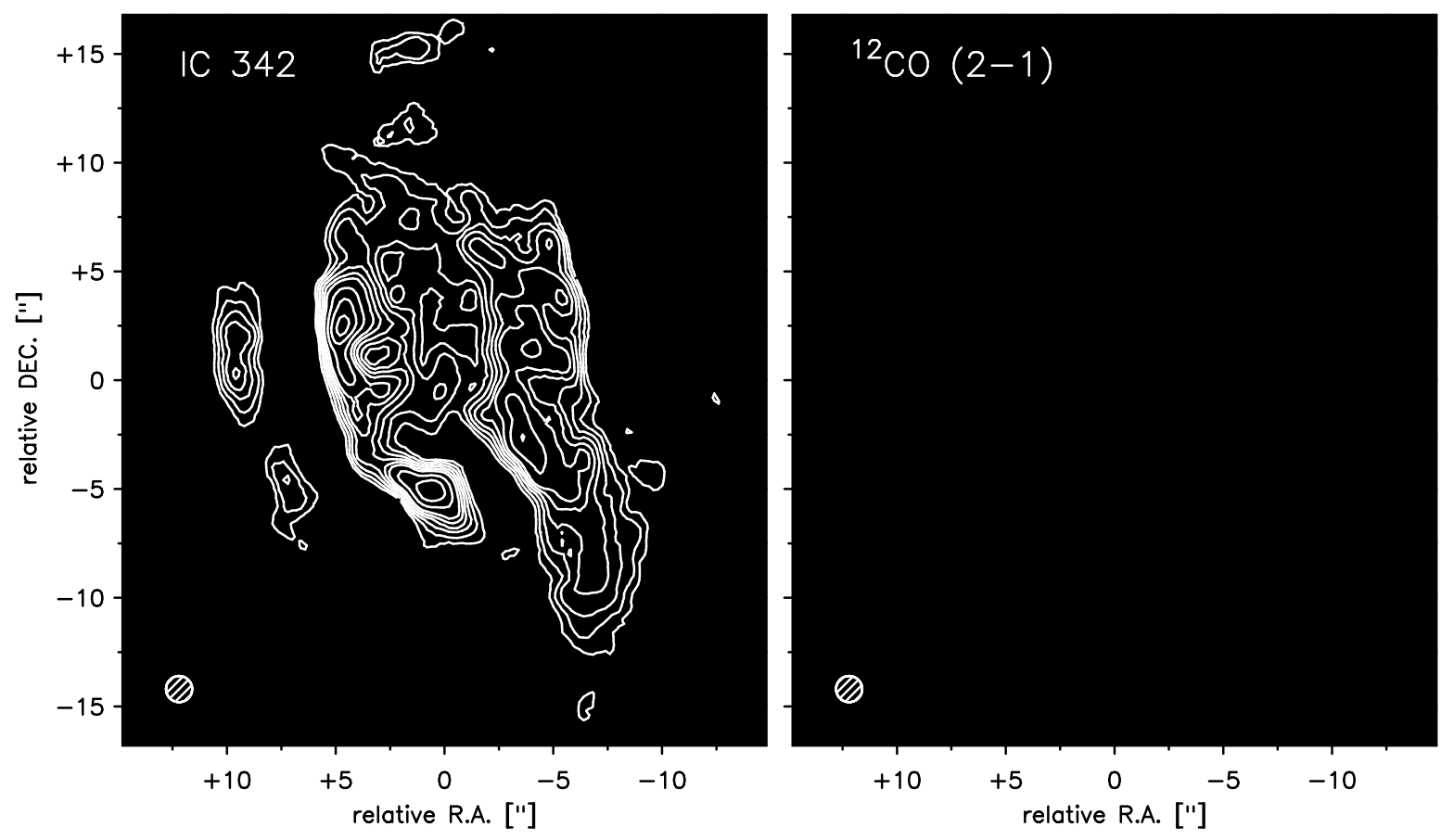

Fig. 1.- OVRO ${ }^{12} \mathrm{CO}(2-1)$ intensity map (left) and velocity field (right) at $10 \mathrm{pc}\left(1.2^{\prime \prime}\right)$ resolution. The contours in the intensity map are at 4, 6, 8, 10, 12, 14, 18, 22, and $26 \sigma$ $\left(1 \sigma=2.6 \mathrm{Jy}_{\mathrm{beam}}{ }^{-1} \mathrm{~km} \mathrm{~s}^{-1}\right)$. The coordinate center is at 03:46:48.26, +68:05:47.8 (J2000). The beam size is shown in the lower left corner. 


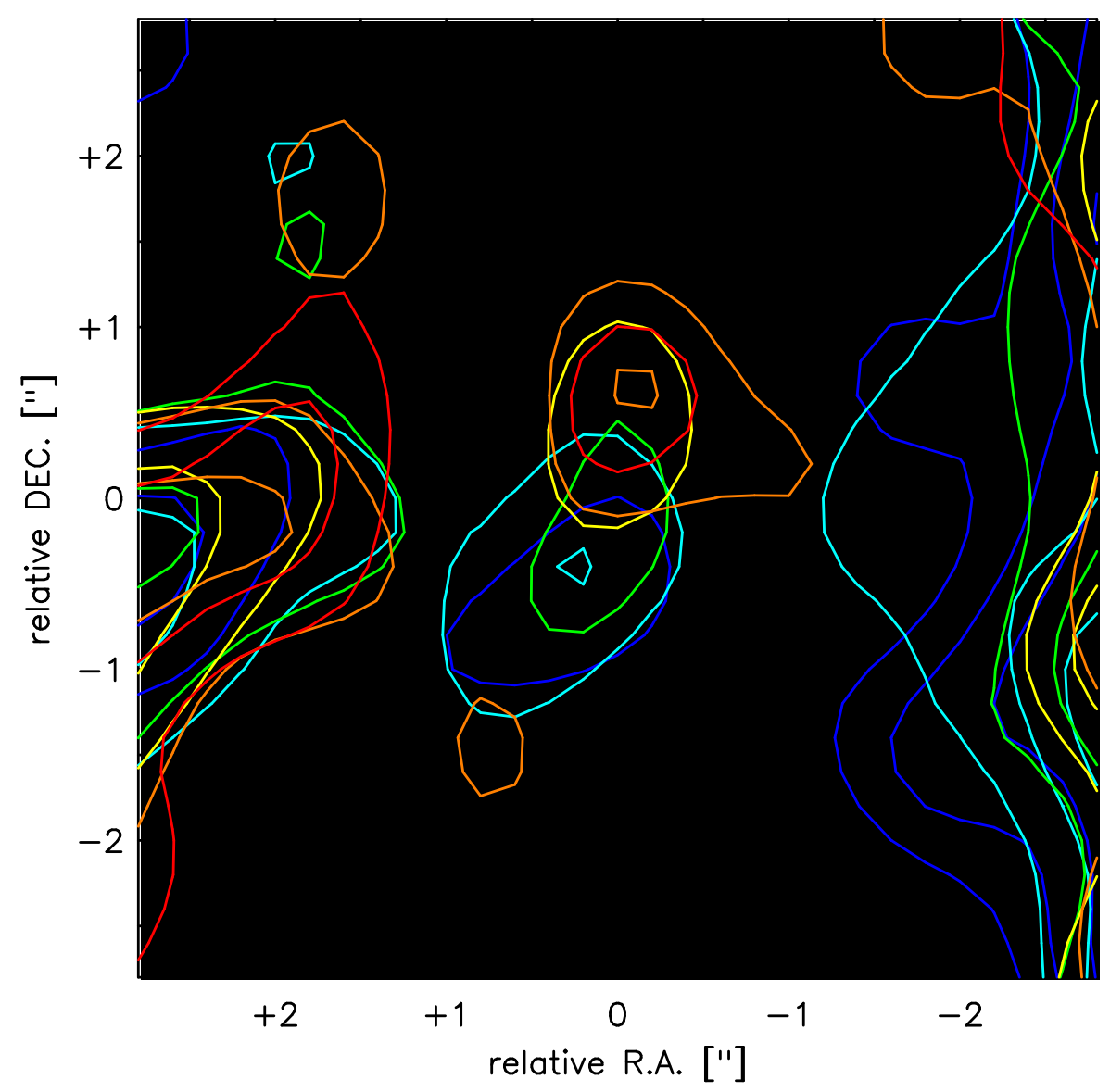

Fig. 2.- Renzogram of ${ }^{12} \mathrm{CO}(2-1)$ emission. Close to the nucleus, the gas is moving along a position angle of $\sim-10^{\circ}$. The contours represent different channel maps from $41.523 \mathrm{~km} \mathrm{~s}^{-1}$ (blue) to $54.528 \mathrm{~km} \mathrm{~s}^{-1}$ (red) in steps of $2.601 \mathrm{~km} \mathrm{~s}^{-1}$. The contours for each channel map are at $15 \sigma, 20 \sigma$ and $25 \sigma$ with $\sigma=40 \mathrm{mJy}$ per beam. The HST F555W image is shown in gray-scale. 


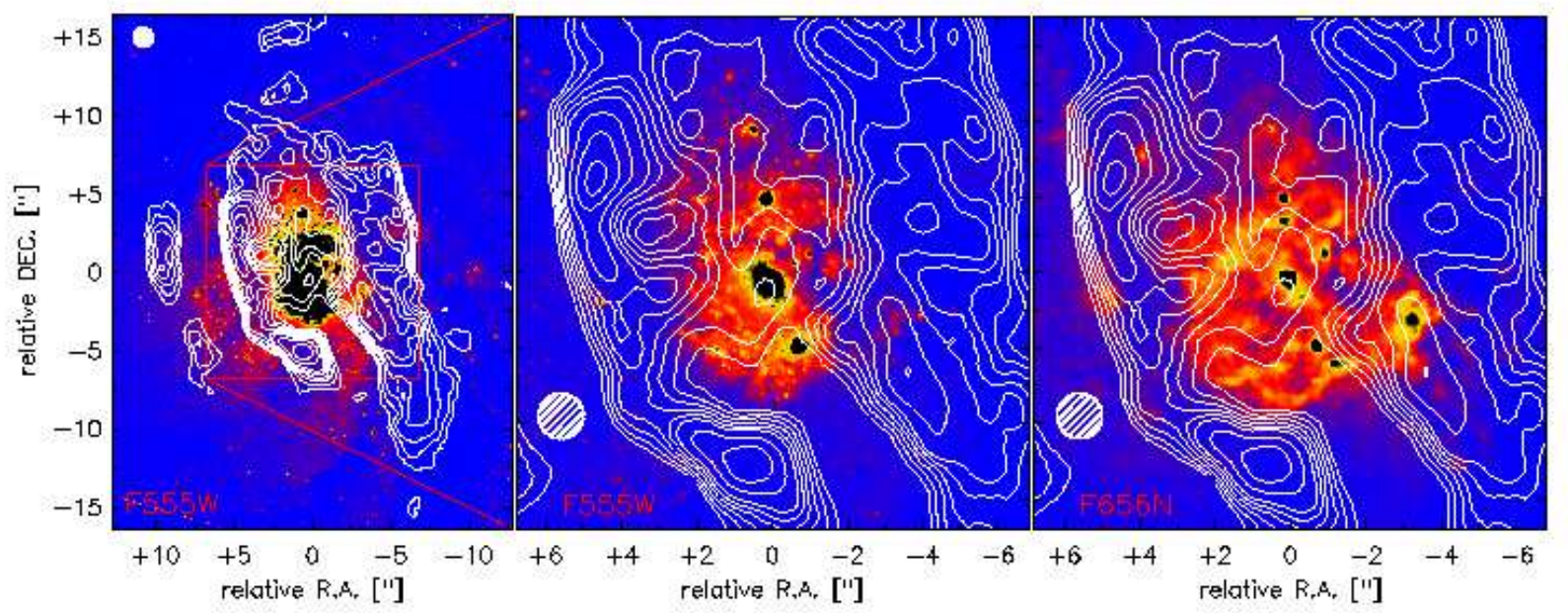

Fig. 3. - The OVRO ${ }^{12} \mathrm{CO}(2-1)$ intensity map overlaid onto the archival HST V-band (left, middle) and $\mathrm{H} \alpha$ image (right). The left panel shows the full OVRO field, while the middle and right panels contain only the inner $120 \mathrm{pc}$ (indicated by a red box in the left panel). Different color stretches are used to enhance low level emission (left) as well as distinct features in the nuclear region (middle) of the HST F555W image. The OVRO CO contours are the same as in Figure 1. For reference, the beam size is shown in each panel. 